\title{
UTILIZAÇÃO DE ANÁLISE ERGOMÉTRICA DE RESULTADOS ESPORTIVOS PARA AVALIAÇÃO DA RESISTÊNCIA ESPECÍFICA DE NADADORES
}

\author{
André Geraldo Brauer Júnior' braverru@hotmail.com \\ Oleg Igorevitch Popov'olegpo57@mail.ru \\ Nina Janovna Bulgakova'
}

doi:10.3900/fpj.7.5.312.p

Braver Júnior AG, Popov OI, Bulgakova NJ. Utilização de análise ergométrica de resultados esportivos para avaliação da resistência específica de nadadores. Fit Pef J. 2008 set-out;7(5):312-8.

\section{RESUMO}

Introdução: Este estudo teve como objetivo analisar a influência do sexo, qualificação esportiva e especialização de distância na resistência específica de nadadores, com a utilização de análise ergométrica de resultados esportivos. Materiais e Métodos: Os coeficientes Kł para avaliação da resistência específica foram calculados de acordo com Gordon (1978), com a divisão do tempo (em segundos) de distâncias adjacentes de natação: $\mathrm{Kt}_{1}=\mathrm{t}_{100} / \mathrm{t}_{50}, \mathrm{Kt}_{2}=\mathrm{t}_{200} / \mathrm{t}_{100}$, $\mathrm{Kt}_{3}=\mathrm{t}_{400} / \mathrm{t}_{200}, \mathrm{Kt}_{4}=t_{1500} / t_{400}$ para homens e $\mathrm{Kt}_{4}=t_{800} / \mathrm{t}_{400}$ para mulheres. Os resultados esportivos foram extraídos da base de dados da Cátedra de Natação da Universidade Estatal de Cultura Física, Esporte e Turismo da Rússia, contendo material relativo a 1295 nadadores russos do estilo crawl (740 homens e 555 mulheres de diferentes qualificações e especializações de distância). Para a avaliação da influência do sexo, especialização de distância e qualificação dos nadadores, utilizou-se a ANOVA para cada um dos coeficientes Kt. Resultados: Os coeficientes para as mulheres situaram-se ligeiramente abaixo dos valores encontrados para os homens, ou seja, as mulheres conseguem manter por mais tempo a velocidade com o aumento da distância. Discussão: $\bigcirc$ nível da resistência analisada através das correlações ergométricas determina a especialização de distância do nadador. Além disso, com o aumento da qualificação esportiva, as diferenças entre os níveis de resistência específica entre os atletas de diferentes distâncias competitivas se tornam mais expressivas.

\section{PALAVRAS-CHAVE}

Sexo, Ergometria, Natação.

'Cátedra de Natação da Universidade Estadual de Cultura Física, Esporte e Turismo da Rússia - Moscou - Rússia

Copyright@ 2008 por Colégio Brasileiro de Atividade Física, Saúde e Esporte

Fit Perf J | Rio de Janeiro | 7 | 5 | 312-318 | set/out 2008 


\title{
USE OF ERGOMETRIC ANALYSIS OF SPORTS RESULTS FOR EVALUATION OF SPECIFIC RESISTANCE OF SWIMMERS
}

\section{ABSTRACT}

Introduction: This study aimed to examine the influence of sex, sports qualification and specialization of distance in the specific resistance of swimmers with the use of ergometric analysis of sports results. Materials and Methods: The coefficients Kt for evaluation of specific resistance were calculated according to Gordon (1978), with the division of time (in seconds) of adjacent distances in the swimming: K $+1=+100 /+50, K+2=+200 /$ $\dagger 100, K+3=\uparrow 400 /+200, K+4=\uparrow 1500 /+400$ for men and $K+4=+800 / \uparrow 400$ for women. Sports results were extracted from database of the Department of Swimming of the State University of Physical Culture, Sports and Tourism of Russia. That database contains materials of 1295 Russian's-freestyle swimmers (740 men and 555 women of different qualifications and expertise away). For the evaluation of the influence of gender, specialization of distance and qualification of swimmers was used the ANOVA for each of the coefficients Kt. Results: The coefficients for women are located slightly below the values found for the men, in other words, women can keep for longer time the speed with the increase of distance. Discussion: The level of resistance analyzed through the ergometric correlations determines the specialization in the swimmer's distance. Also, with the increase of sports qualification, the differences between the levels of specific resistance among athletes from different competitive distances become more expressive.

\section{KEYWORDS}

Sex, Ergometry, Swimming.

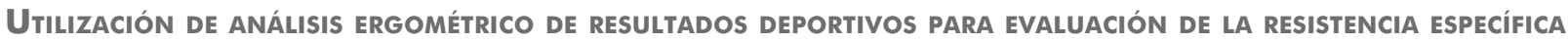 DE NADADORES}

\section{RESUMEN}

Introdución: Este estudio tuvo como objetivo analizar la influencia del sexo, calificación deportiva y especialización de distancia en la resistencia específica de nadadores con la utilización de análisis ergométrico de resultados deportivos. Materiales y Métodos: Los coeficientes Kt para evaluación de la resistencia fueron calculados de acuerdo a Gordon (1978), con la división del tiempo (en segundos) de distancias adyacentes de natación: $\mathrm{K} \uparrow \mathrm{l}=+100 /+50, \mathrm{~K}+2=+200 / \uparrow 100, \mathrm{KT} 3=+400 /+200, \mathrm{~K}+4=+1500 /+400$ para los hombres y $\mathrm{K}+4=+800 / \uparrow 400$ para mujeres. Los resultados deportivos fueron extraídos de la base de datos de la Cátedra de Natación de la Universidad Estatal de Cultura Física, Deportes y Turismo de Rusia, que contenía material relativa a 1295 nadadores rusos de estilo crawl (740 hombres y 555 mujeres de diferentes calificaciones y especializaciones de distancia). Para la evaluación de la influencia del sexo, especialización de distancia y calificación de los nadadores se utilizó ANOVA para cada uno de los coeficientes Kt. Resultados: Los coeficientes para las mujeres se sitúan ligeramente por abajo de los valores encontrados para los hombres, o sea, las mujeres consiguen mantener por más tiempo la velocidad con el aumentó de la distancia. Discusión: El nivel de resistencia analizada a través de las correlaciones ergométricas determina la distancia la especialización de distancia del nadador, también, con el aumento de la calificación deportiva las diferencias entre los niveles de resistencia específica entre los atletas de diferentes distancias competitivas se convierten más expresivas.

\section{PALABRAS CLAVE}

\author{
Sexo, Ergometría, Natación.
}

\section{INTRODUÇÃO}

Os resultados do nadador na sua distância principal estão estreitamente relacionados com o nível dos resultados em distâncias adjacentes, ou seja, para o sucesso em provas de velocidade é necessário possuir determinado nível de resistência em diferentes zonas de potência, inclusive na zona aeróbica. Uma insuficiente velocidade e resistência de velocidade pode ser um grande obstáculo para o posterior crescimento dos resultados de nadadores fundistas, sendo assim, em nadadores fundistas ou velocistas deve existir um balanço ótimo de desenvolvimento das capacidades aeróbica e anaeróbica. A avaliação integral do nível de desenvolvimento da resistência em diferentes zonas de potência pode ser obtida através da construção de relações ergométricas do tipo: distância-tempo, velocidade-tempo ou análogos.

Frequentemente, na qualidade de dados iniciais para a análise ergométrica são utilizados os recordes mundiais ${ }^{\mathbf{1 , 2 , 3 , 4}}$ ou resultados ergométricos em testes ${ }^{\mathbf{5 , 6 , 7 , 8 , 9}}$. Sendo que a curva dos recordes está fundamentado nas principais leis médico-biológicas, então esta análise pode ser aplicada para os resultados esportivos. Na natação esportiva a análise ergométrica dos resultados é descrita por Gordon \& Morozov ${ }^{\mathbf{1 0}}$, Vanikov ${ }^{11}$ e Naumenko ${ }^{\mathbf{1 2}}$.

Na prática esportiva, são amplamente utilizados coeficientes de resistência mais simples e visíveis como, por exemplo, o coeficiente de utilização, que é calculado como sendo o percentual da velocidade em dada distância em relação à distância de 100m (por vezes, leva-se em consideração como

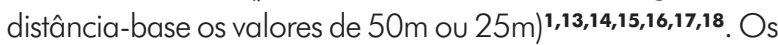
resultados esperados em distâncias complementares, por vezes é obtido através de equações de regressão linear ${ }^{11,19,20}$. $\mathrm{Na}$ literatura existem diferentes variantes de coeficientes que, de uma forma ou de outra, avaliam a reserva de velocidade ou resistência de atletas em esportes cíclicos ${ }^{21,22,23}$. 
Gordon \& Morozov ${ }^{\mathbf{1 0} 24}$ sugeriram os coeficientes Kt, que são calculados como sendo as partes da divisão do tempo (em segundos) de passagem em distâncias adjacentes de natação (200m e 100m, 400m e 200m e assim por diante). Esses coeficientes indicam o nível da resistência específica em diferentes distâncias e os resultados esperados para cada uma delas. Apesar da grande quantidade de trabalhos que descrevem uma ou outra forma de obtenção dos resultados esperados em distâncias adjacentes, somente nos trabalhos de Gordon ${ }^{10}$ são apresentadas relações ergométricas que caracterizam a velocidade relativa de nadadores do estilo crawl de diferentes qualificações e especializações de distância. Contudo, estes resultados foram obtidos apenas na base dos resultados de atletas do sexo masculino.

Sendo assim, o objetivo do presente estudo é analisar a influência do sexo, qualificação esportiva e especialização de distância na resistência específica de nadadores, através da utilização de análise ergométrica de resultados esportivos.

\section{MATERIAIS E MÉTODOS}

\section{Aprovação do estudo}

Oestudo foi aprovado pelo Comitê de Ética em Pesquisa da Universidade Estatal de Cultura Física, Esporte e Turismo da Rússia - Moscou, e atende a todas as normas do Conselho Nacional de Saúde de 10/10/1996.

\section{Sujeitos}

Os valores normativos para o coeficiente $\mathrm{Kt}$ foram obtidos através da elaboração dos melhores resultados esportivos de 1295 nadadores russos do estilo crawl (740 homens e 555 mulheres de diferentes qualificações esportivas e especializações de distância). Para tanto, consultou-se a base de dados da Cátedra de Natação da Universidade Estatal de Cultura Física, Esporte e Turismo da Rússia, que contém material relativo aos melhores resultados esportivos de nadadores russos de diferentes qualificações esportivas e

Tabela 1 - Valores dos coeficientes $\mathrm{K}_{\dagger}\left(\bar{X}_{ \pm \sigma}\right.$ ) para nadadores do estilo crawl de diferentes qualificações esportivas, especialistas nas distâncias de $50 \mathrm{~m}$ a $1500 \mathrm{~m}(\mathrm{n}=740)$

\begin{tabular}{|c|c|c|c|c|}
\hline Qualificação & $\mathrm{Kt}_{1}=\mathrm{t}_{100} / \mathrm{t}_{50}$ & $\mathrm{Kt}_{2}=\mathrm{t}_{200} / \mathrm{t}_{100}$ & $\mathrm{Kt}_{3}=\mathrm{t}_{400} / \mathrm{t}_{200}$ & $\mathrm{Kt}_{4}=\mathrm{t}_{1500} / \mathrm{t}_{4}$ \\
\hline \multicolumn{5}{|c|}{ Especialistas na distância de $50 \mathrm{~m}$} \\
\hline II & $2,2324 \pm 0,0268$ & $2,2289 \pm 0,0266$ & $2,1510 \pm 0,0332$ & $4,0318 \pm 0,0785$ \\
\hline I & $2,2311 \pm 0,0215$ & $2,2400 \pm 0,0262$ & $2,1516 \pm 0,0319$ & $4,0839 \pm 0,0662$ \\
\hline CME & $2,2408 \pm 0,0198$ & $2,2369 \pm 0,0201$ & $2,1784 \pm 0,0217$ & $4,0996 \pm 0,0498$ \\
\hline ME & $2,2498 \pm 0,0199$ & $2,2347 \pm 0,0292$ & $2,1752 \pm 0,0224$ & $4,1094 \pm 0,0476$ \\
\hline MENI & $2,2525 \pm 0,0242$ & $2,2520 \pm 0,0265$ & $2,1950 \pm 0,0207$ & $4,1292 \pm 0,0420$ \\
\hline \multicolumn{5}{|c|}{ Especialistas na distância de $100 \mathrm{~m}$} \\
\hline II & $2,1888 \pm 0,0286$ & $2,2127 \pm 0,0216$ & $2,1365 \pm 0,0313$ & $4,0241 \pm 0,0487$ \\
\hline I & $2,1802 \pm 0,0322$ & $2,2139 \pm 0,0247$ & $2,1488 \pm 0,0375$ & $4,0391 \pm 0,0553$ \\
\hline CME & $2,1748 \pm 0,0334$ & $2,2239 \pm 0,0277$ & $2,1612 \pm 0,0425$ & $4,0512 \pm 0,0582$ \\
\hline ME & $2,1718 \pm 0,0178$ & $2,2203 \pm 0,0223$ & $2,1771 \pm 0,0229$ & $4,0791 \pm 0,0511$ \\
\hline MENI & $2,1774 \pm 0,0185$ & $2,2243 \pm 0,0317$ & $2,1863 \pm 0,0267$ & $4,1070 \pm 0,0556$ \\
\hline \multicolumn{5}{|c|}{ Especialistas na distância de $200 \mathrm{~m}$} \\
\hline II & $2,1760 \pm 0,0246$ & $2,1581 \pm 0,0305$ & $2,1326 \pm 0,0259$ & $4,0044 \pm 0,0591$ \\
\hline I & $2,1714 \pm 0,0295$ & $2,1608 \pm 0,0235$ & $2,1383 \pm 0,0225$ & $4,0151 \pm 0,0526$ \\
\hline CME & $2,1684 \pm 0,0321$ & $2,1549 \pm 0,0243$ & $2,1425 \pm 0,0161$ & $4,0399 \pm 0,0760$ \\
\hline ME & $2,1592 \pm 0,0322$ & $2,1549 \pm 0,0318$ & $2,1475 \pm 0,0302$ & $4,0549 \pm 0,0442$ \\
\hline MENI & $2,1501 \pm 0,0227$ & $2,1567 \pm 0,0231$ & $2,1494 \pm 0,0225$ & $4,0772 \pm 0,0580$ \\
\hline \multicolumn{5}{|c|}{ Especialistas na distância de $400 \mathrm{~m}$} \\
\hline II & $2,1679 \pm 0,0308$ & $2,1571 \pm 0,0361$ & $2,0899 \pm 0,0272$ & $3,9711 \pm 0,0670$ \\
\hline 1 & $2,1665 \pm 0,0389$ & $2,1571 \pm 0,0360$ & $2,0836 \pm 0,0224$ & $4,0046 \pm 0,0688$ \\
\hline CME & $2,1663 \pm 0,0268$ & $2,1522 \pm 0,0325$ & $2,0841 \pm 0,0216$ & $4,0078 \pm 0,0583$ \\
\hline ME & $2,1559 \pm 0,0310$ & $2,1459 \pm 0,0294$ & $2,0795 \pm 0,0258$ & $4,0240 \pm 0,0578$ \\
\hline MENI & $2,1411 \pm 0,0212$ & $2,1324 \pm 0,0247$ & $2,0952 \pm 0,0222$ & $4,0212 \pm 0,0599$ \\
\hline \multicolumn{5}{|c|}{ Especialistas na distância de 1500m } \\
\hline II & $2,1590 \pm 0,0329$ & $2,1452 \pm 0,0303$ & $2,0839 \pm 0,0280$ & $3,9378 \pm 0,0779$ \\
\hline I & $2,1527 \pm 0,0321$ & $2,1462 \pm 0,0277$ & $2,0824 \pm 0,0194$ & $3,9238 \pm 0,0542$ \\
\hline CME & $2,1520 \pm 0,0280$ & $2,1350 \pm 0,0222$ & $2,0762 \pm 0,0266$ & $3,9128 \pm 0,0545$ \\
\hline ME & $2,1502 \pm 0,0206$ & $2,1258 \pm 0,0322$ & $2,0729 \pm 0,0250$ & $3,9041 \pm 0,0527$ \\
\hline MENI & $2,1328 \pm 0,0106$ & $2,1103 \pm 0,0281$ & $2,0635 \pm 0,0248$ & $3,8810 \pm 0,0574$ \\
\hline
\end{tabular}


especializações. Na Rússia, os atletas são condicionalmente divididos pelas seguintes categorias: a - atletas de III categoria; b - atletas de II categoria; c - atletas de I categoria; $d$ - candidatos à mestre do esporte (CME); e - mestres do esporte (ME); $f$ - mestres do esporte de nível internacional (MENI). As categorias que vão desde a III até ME são atingidas através do alcance de resultados esportivos normativos. Já para receber a denominação de MENI, é necessário alcançar determinados resultados esportivos em Jogos Olímpicos e campeonatos mundiais.

\section{Determinação dos coeficientes Kt}

O coeficiente Kt é utilizado para avaliar, de forma indireta, a resistência específica do nadador ou sua capacidade de manter a velocidade com o aumento da distância, sendo que, quanto menores forem os valores de Kt, menos o atleta perde velocidade com o aumento da distância, indicando assim a sua resistência para dado trabalho. Os coeficientes Kt foram calculados, de acordo com Gordon ${ }^{10}$, como sendo a divisão do tempo (em segundos) de distâncias adjacentes de natação: $\mathrm{K}_{1}=\mathrm{t}_{100} / \mathrm{t}_{50}$ $\mathrm{Kt}_{2}=\mathrm{t}_{200} / \mathrm{t}_{100 ;} \mathrm{Kt}_{3=} \mathrm{t}_{400} / \mathrm{t}_{200 ;}$ sendo $\mathrm{Kt}_{4}=\mathrm{t}_{1500} / \mathrm{t}_{400}$ para homens $\mathrm{e} \mathrm{Kt}_{4}=\mathrm{t}_{800} / \mathrm{t}_{400}$ para mulheres. $\mathrm{O}$ coeficiente $\mathrm{Kt}_{1}$ caracteriza a resistência de velocidade, $\mathrm{Kt}_{2}$ caracteriza a resistência em exercícios anaeróbicos glicolíticos, $\mathrm{Kt}_{3}$ caracteriza a resistência em distâncias de meio-fundo e $\mathrm{Kt}_{4}$ caracteriza a resistência em distâncias de fundo. Além disso, com o auxílio dos valores médios de K† (Tabelas 1 e 2) é possível calcular os resultados esperados em distâncias adjacentes. Se o nadador apresenta resultados próximos aos calculados, então a sua resistência na distância correspondente se encontra em um nível suficiente. Se os resultados apresentados situam-se ligeiramente abaixo dos esperados, então é necessário introduzir algumas correções no processo de treinamento. Já uma diferença grande entre os resultados esperados e os apresentados está relacionada à preparação insuficiente na distância fundamental, podendo indicar que o atleta deve mudar de especialização.

Tabela 2 - Valores dos coeficientes $K_{\dagger}\left(\bar{X}_{ \pm \sigma)}\right.$ para nadadoras do estilo crawl de diferentes qualificações esportivas, especialistas nas distâncias de $50 \mathrm{~m}$ a $800 \mathrm{~m}(\mathrm{n}=555)$

\begin{tabular}{|c|c|c|c|c|}
\hline Qualificação & $\mathrm{Kt}_{1}=\mathrm{t}_{100} / \mathrm{t}_{50}$ & $\mathrm{Kt}_{2}=\mathrm{t}_{200} / \mathrm{t}_{100}$ & $\mathrm{Kt}_{3}=\mathrm{t}_{400} / \mathrm{t}_{200}$ & $\mathrm{Kt}_{4}=\mathrm{t}_{800} / \mathrm{t}_{400}$ \\
\hline \multicolumn{5}{|c|}{ Especialistas na distância de $50 \mathrm{~m}$} \\
\hline II & $2,2162 \pm 0,0180$ & $2,2052 \pm 0,0190$ & $2,1196 \pm 0,0193$ & $2,1036 \pm 0,0237$ \\
\hline I & $2,2190 \pm 0,0216$ & $2,2076 \pm 0,0234$ & $2,1234 \pm 0,0251$ & $2,1072 \pm 0,0261$ \\
\hline CME & $2,2214 \pm 0,0238$ & $2,2123 \pm 0,0259$ & $2,1362 \pm 0,0267$ & $2,1148 \pm 0,0259$ \\
\hline ME & $2,2275 \pm 0,0264$ & $2,2170 \pm 0,0297$ & $2,1459 \pm 0,0321$ & $2,1162 \pm 0,0263$ \\
\hline MENI & $2,2306 \pm 0,0326$ & $2,2261 \pm 0,0471$ & $2,1509 \pm 0,0245$ & $2,1353 \pm 0,0258$ \\
\hline \multicolumn{5}{|c|}{ Especialistas na distância $100 \mathrm{~m}$} \\
\hline II & $2,1802 \pm 0,0186$ & $2,1722 \pm 0,0208$ & $2,1024 \pm 0,0226$ & $2,0783 \pm 0,0144$ \\
\hline I & $2,1753 \pm 0,0152$ & $2,1818 \pm 0,0260$ & $2,1052 \pm 0,0223$ & $2,0855 \pm 0,0184$ \\
\hline CME & $2,1771 \pm 0,0163$ & $2,1736 \pm 0,0176$ & $2,1093 \pm 0,0232$ & $2,0762 \pm 0,0215$ \\
\hline ME & $2,1751 \pm 0,0216$ & $2,1802 \pm 0,0239$ & $2,1210 \pm 0,0254$ & $2,0913 \pm 0,0222$ \\
\hline MENI & $2,1683 \pm 0,0151$ & $2,1832 \pm 0,0242$ & $2,1263 \pm 0,0194$ & $2,1025 \pm 0,0202$ \\
\hline \multicolumn{5}{|c|}{ Especialistas na distância $200 \mathrm{~m}$} \\
\hline II & $2,1840 \pm 0,0341$ & $2,1483 \pm 0,0258$ & $2,0965 \pm 0,0249$ & $2,0726 \pm 0,0244$ \\
\hline I & $2,1736 \pm 0,0337$ & $2,1282 \pm 0,0198$ & $2,1113 \pm 0,0292$ & $2,0688 \pm 0,0207$ \\
\hline CME & $2,1681 \pm 0,0364$ & $2,1355 \pm 0,0159$ & $2,0958 \pm 0,0181$ & $2,0668 \pm 0,0191$ \\
\hline ME & $2,1558 \pm 0,0205$ & $2,1265 \pm 0,0293$ & $2,1104 \pm 0,0292$ & $2,0855 \pm 0,0283$ \\
\hline MENI & $2,1317 \pm 0,0177$ & $2,1266 \pm 0,0174$ & & $2,0767 \pm 0,0141$ \\
\hline \multicolumn{5}{|c|}{ Especialistas na distância $400 \mathrm{~m}$} \\
\hline II & $2,1588 \pm 0,0213$ & $2,1420 \pm 0,0347$ & $2,0849 \pm 0,0297$ & $2,0640 \pm 0,0232$ \\
\hline I & $2,1506 \pm 0,0190$ & $2,1415 \pm 0,0213$ & $2,0781 \pm 0,0205$ & $2,0610 \pm 0,0193$ \\
\hline CME & $2,1481 \pm 0,0226$ & $2,1300 \pm 0,0262$ & $2,0734 \pm 0,0202$ & $2,0596 \pm 0,0183$ \\
\hline ME & $2,1392 \pm 0,0351$ & $2,1191 \pm 0,0286$ & $2,0744 \pm 0,0217$ & $2,0569 \pm 0,0171$ \\
\hline MENI & $2,1120 \pm 0,0168$ & $2,1036 \pm 0,0166$ & $2,0837 \pm 0,0199$ & $2,0631 \pm 0,0191$ \\
\hline \multicolumn{5}{|c|}{ Especialistas na distância $800 \mathrm{~m}$} \\
\hline II & $2,1460 \pm 0,0279$ & $2,1341 \pm 0,0271$ & $2,0786 \pm 0,0211$ & $2,0570 \pm 0,0244$ \\
\hline I & $2,1461 \pm 0,0231$ & $2,1244 \pm 0,0264$ & $2,0754 \pm 0,0202$ & $2,0475 \pm 0,0172$ \\
\hline CME & $2,1356 \pm 0,0293$ & $2,1189 \pm 0,0338$ & $2,0647 \pm 0,0257$ & $2,0412 \pm 0,0216$ \\
\hline ME & $2,1145 \pm 0,0310$ & $2,1037 \pm 0,0292$ & $2,0553 \pm 0,0201$ & $2,0396 \pm 0,0221$ \\
\hline MENI & $2,0845 \pm 0,0231$ & $2,0824 \pm 0,0228$ & $2,0410 \pm 0,0225$ & $2,0331 \pm 0,0136$ \\
\hline
\end{tabular}




\section{Análise estatística}

Para avaliação quantitativa da influência do sexo, especialização de distância e qualificação do nadador em cada um dos coeficientes Kt, foi realizada análise de dispersão ANOVA com três fatores: sexo; qualificação esportiva; e especialização de distância. $\bigcirc$ critério de Fisher foi utilizado com o objetivo de comparar as diferenças entre as dispersões. $\bigcirc$ nível de significância adotado foi de $p \leq 0,05$. Além disso, foi utilizada a média e o desvio-padrão para a distribuição de cada um dos valores Kt, de acordo com a especialização na distância. Utilizou-se o programa Statistica 6.0 para a análise dos dados.

\section{RESULTADOS}

Nas Tabelas 1 e 2 e nos Gráficos 1 a 8, são representados os valores médios para os diferentes coeficientes $\mathrm{K} \dagger$ de nadadores e nadadoras especializados em diferentes distâncias, com qualificação esportiva variando de II categoria até mestre do esporte de nível internacional. Percebe-se que o sexo, a especialização e a qualificação do nadador influenciam na estrutura da preparação para determinada distância. Para os coeficientes Kł1, Kł2 e Kł3 houve influência significativa de cada um dos fatores $(F>5,73, p<0,000034)$, e também a interação: 1) sexo e especialização $(F>2,69, p<0,03)$; 2) qualificação e especialização $\left(F>4,90, p<7,37 \times 10^{-10}\right)$. Influência mútua do sexo e qualificação foi observada para os coeficientes K†1 e K†3, já a interação simultânea de todos os três fatores não foi estatisticamente significativa.

Praticamente, todos os valores do coeficiente $\mathrm{Kt}$ para as mulheres se situam ligeiramente abaixo dos valores encontrados para os homens, o que significa que as mulheres apre-

Gráficos 1, 2, 3 e 4 - Valores dos coeficientes Kt para nadadores de diferentes qualificações esportivas (a partir de II categoria até MENI) e especializações de distância (de $50 \mathrm{~m}$ a 1500m), n=740
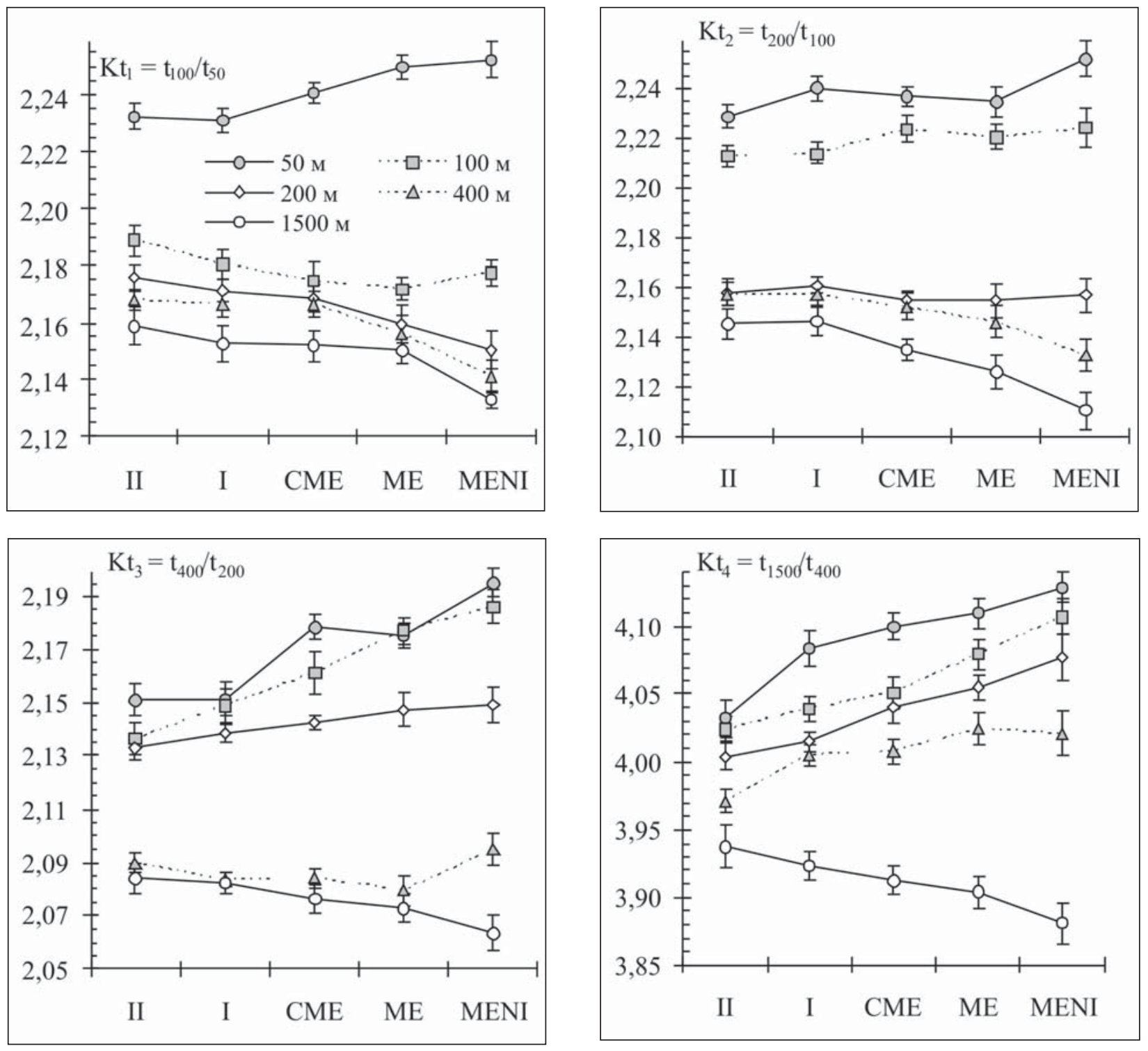
sentam uma maior resistência relativa, ou seja, conseguem manter a velocidade por maior tempo com o aumento da distância. No geral, a influência do sexo nos valores ergométricos dos coeficientes se mostra nítida. No entanto, em separado, diante da comparação pareada Kł para a correspondente especialização e qualificação, apenas em 1/3 dos casos as diferenças foram estatisticamente significativas.

\section{DISCUSSÃO}

fator de maior importância que determina a resistência relativa do nadador é representado pela especialização na distância. Em maior grau, diferenciam-se os coeficientes Kt que determinam a resistência em distâncias próximas da distância competitiva fundamental. Sendo assim, em nadadores ME e MENI, especialistas nas distâncias de $50 \mathrm{~m}$ e $100 \mathrm{~m}$, a diferença nos coeficientes $\mathrm{K} \nmid l$ é significativa; assim como para os coeficientes $\mathrm{K} \dagger 4$ para esses atletas, as diferenças não são significativas. Nos nadadores em que as distâncias competitivas fundamentais diferenciam-se significativamente (por exemplo, $100 \mathrm{~m}$ e $400 \mathrm{~m}$ ), existe uma nítida diferença no nível de preparação especial para dada distância. No entanto, se compararmos os nadadores especializados em distâncias adjacentes (100m e 200m, ou 200m e 400m), as diferenças são nitidamente menores, o que explica o fato de que muitos nadadores competem nessas distâncias com grande sucesso. Por outro lado, os velocistas que competem na distância de $50 \mathrm{~m}$, visivelmente dividem-se em um grupo à parte.

Gráficos 5, 6, 7 e 8 - Valores dos coeficientes Kł para nadadoras de diferentes qualificações esportivas (a partir de II categoria até $\mathrm{MENI}$ ) e especializações de distância (de $50 \mathrm{~m}$ a $800 \mathrm{~m}$ ), $\mathrm{n}=555$
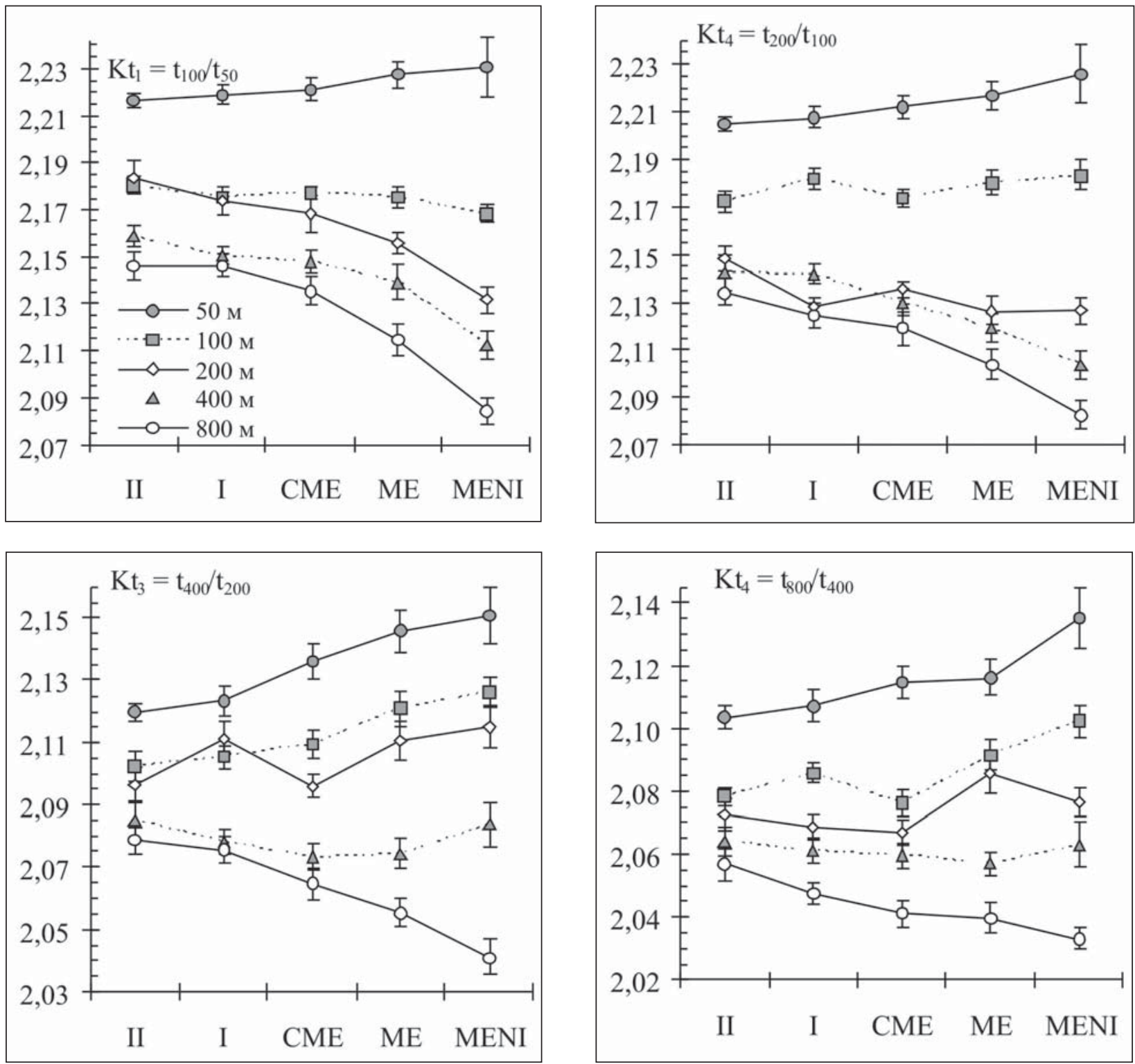
À medida que a maestria esportiva vai aumentando, se torna mais expressiva a especialização em determinada distância: em velocistas das provas de $50 \mathrm{~m}$ e $100 \mathrm{~m}$, com o aumento da qualificação a resistência relativa diminui praticamente em todas as zonas fisiológicas de potência, enquanto que, em fundistas, ela praticamente aumenta continuamente (os valores de todos os coeficientes Kt diminuem).

Os coeficientes Kt que caracterizam o nível de preparação dos nadadores pela distância é suficientemente estável em atletas de diferentes gerações. Em comparação realizada com os dados de Morozov ${ }^{24}$, em nadadores da década de 90, foi detectada uma fraca tendência de aumento da reserva de velocidade (aumento do Kt). No entanto, na grande maioria dos casos, ela não é estatisticamente significativa. A exceção são os nadadores especialistas na distância de 100m, nos quais o coeficiente Kt diminuiu, significando um aumento da resistência relativa. Isso pode ser explicado pelo fato de que foi introduzida uma nova distância competitiva $(50 \mathrm{~m})$ e, de uma forma mais nítida, os velocistas começaram a se preparar de forma mais direcionada para essa distância. Nesses atletas observam-se os maiores valores para todos os coeficientes $\mathrm{Kt}$.

O nível de preparação pela distância, dos campeões dos últimos Jogos Olímpicos, corrobora os dados mostrados. Os resultados apresentados por Pieter Hoogenband $(22,03 \mathrm{~s}, 47,84 \mathrm{~s}$ e $1 \min 45,35 \mathrm{~s}$ nas distâncias de $50 \mathrm{~m}$, $100 \mathrm{~m}$ e $200 \mathrm{~m}$ estilo livre) com os cálculos do Coeficiente Kt1 $(2,1716)$ e Kt $2(2,2021)$, estão em concordância com os valores K† para nadadores MENI, especialistas na distância de $100 \mathrm{~m}(2,1774 \pm 0,0185$ e 2,2243 $\pm 0,0317$, respectivamente). $\mathrm{O}$ coeficiente $\mathrm{K}+3$ calculado para os resultados de lan Torpe $(1 \mathrm{~min} 45,37 \mathrm{~s}$ e $3 \mathrm{~min} 40,59 \mathrm{~s}$ nas distâncias de $200 \mathrm{~m}$ e $400 \mathrm{~m}$ ) também se encontram dentro dos intervalos de valores para nadadores MENI, para nadadores especialistas na distância de $400 \mathrm{~m}$ $(2,0952 \pm 0,0222)$.

O nível de resistência relativa de nadadores avaliados com a utilização de análises ergométricas determina, em um grau muito elevado, a distância de especialização do nadador. Em menor grau, o nível de preparação em dada distância depende do sexo e qualificação do atleta.

Com o aumento da qualificação dos nadadores, acentuam-se as diferenças entre os níveis de resistência específica dos atletas nas diferentes distâncias competitivas.

Nos últimos 10-15 anos não ocorreram mudanças significativas nos parâmetros das correlações ergométricas, determinantes da reserva de velocidade e resistência dos nadadores. Isto nos permite recomendar os dados apresentados na qualidade de modelos característicos.

Comparando os valores reais com os adequados, é possível determinar possíveis insuficiências na preparação específica do nadador, bem como indicar com precisão a distância de especialização do atleta.

\section{REFERÊNCIAS}

1. Ettema JE. Limits of Human Performance and Energy production. Int $Z$ Angew Physiol Einschl Arbeitphysiol. 1966;22:45-54.

2. Henry FM. Prediction of world records in running sixty yards to twenty-six miles. Res Q. 1955;26:147-58.

3. Lloyd BB. The energetics of running: an analysis of world records. Adv Sci. 1966;22(103):515-30.

4. Mognoni P, Lafontuna C, Russo G, Minetti A. An analysis of world records in three types of locomotion. Eur J Appl Physiol. 1982;49:287-99.

5. Monod H, Scherrer J. The work capacity of a synergic muscular group. Ergonomics. 1965;8:329-38.

6. Moritani T, Nagata A, De Vries HA, Muro M. Critical power as a measure of physical work capacity and anaerobic threshold. Ergonomics. $1981 ; 24: 339-50$.

7. Morton RH, Hodgson DJ. The relationship between power output and endurance: a brief review. Eur J Appl Physiol. 1996;73:491-502.

8. Vandewalle H. Ergometrie. In: Biologie de I'exercice musculaire. Paris: Masson; 1992.

9. Vandewalle H, Vautier JF, Kachouri M, Lechevalier JM, Monod H. Workexhaustion time relationships and the critical power concept. A critical review. J Sports Med Phys Fitness. 1997;37:89-102.

10. Gordon SM, Morozov SN. Ispolzovanie analiza rezultatov v upravlenii trenirovkoi plovtsa. In: Plavanie. Moskva: Sportivnaia; 1978.

11. Banikov AA. Optimizatsia podgotovki sportivnovo rezerva na osnavanii analiza mnogoletnei trenirovki plovtsov vuisokovo klassa. Teoria i praktika fizitcheskoi kulturii. Moskva; 1978.

12. Naumenko VK. Ergometritcheskii analiz rekordov mira i URSS po plavaniu. In: Plavanie. Moskva: Sportivnaia; 1978

13. Konov SP. Efektivnie metodii pedagogitcheskovo kontrolia v soverchenstvovanii fizitcheskoi podgotovlenosti iunikh plovtsov. Teoria i praktika fizitcheskoi kulturii. Moskva; 1982.

14. Nabatnikova M. O diapazone «distantsionoi» podgotovlenosti plovtsov. In: Plavanie. Moskva: Sportivnaia; 1974.

15. Nabatnikova M, Ivochkin VV. Sistema kompleksnovo kontrolia v upravlenii podgotovkoi iunikh sportsmenov. Moskva: Olimpiskaia; 1982.

16. Petukhov SI. Struktura trenirovotchinikh nagruzok na razlichnuikh etapakh mnogoletnei podgotovki plovtsov 9-14 let. Teoria i praktika fizitcheskoi kulturii. Moskva; 1984

17. Platonov VN, Bulatova ML. Kontrol vuinoslivosti sportsmena. Kiev: KGIFK; 1992.

18. Platonov VN, Vaitsekhovski SM. Trenirovka plovtsov buisokovo klassa. Moskva: Fizkultura i Sport; 1985.

19. Bulgakova NJ, Banikova JS, Banikov AA. Organizatsia i metodika podgotovki sportivnovo rezerva v plavanii. Moskva: GTSOLIFK; 1982

20. Kuzovenkov VV, Makaruitchev VA. Metodui povuichenia rezultativnosti plovtsov na distantsiakh 50m i 100m volinuim stilem. Plavanie. 1988;10:1623.

21. Zatsiorski VM, Alechinskii SI, lakunin NA. Biomekhanicheskie osnobui vuinoslivost. Moskva: Fizkultura i sport; 1982.

22. Zatsiorski VM, Volkov NI, Kulik NG. Odvukh tipakh pokazatelei buinoslivost. Teoria i praktika fizitcheskoi kulturui. 1965;2:21-5

23. Zemliakov VE. K voprosi opredelenia rabotospocobnosti i spetsialnoi buinoslivosti $v$ tsiklitcheskikh vidakh sporta. Teoria i praktika fizitcheskoi kulturui. Moskva; 1990.

24. Morozov SN. Otsenka sostoiania fizitcheskoi podgotovlenosti plovtsov sprinterov $\mathrm{i}$ staierov $\mathrm{v}$ sisteme upravlenia trenirovochnim protsesom. Moskva: Gtsolifk; 1983.

Recebido: 02/06/2008 - Aceito: 16/09/2008 